ITP-UU-07/9, SPIN-07/9

\title{
Vacuum properties of nonsymmetric gravity in de Sitter space
}

\author{
Tomas Janssen* and Tomislav Prokoped \\ Institute for Theoretical Physics (ITP) 8 Spinoza Institute, Utrecht University, \\ Leuvenlaan 4, Postbus 80.195, 3508 TD Utrecht, The Netherlands
}

\begin{abstract}
We consider quantum effects of a massive antisymmetric tensor field on the dynamics of de Sitter spacetime. Our starting point is the most general, stable, linearized Lagrangian arising in nonsymmetric gravitational theories (NGTs), where part of the antisymmetric field mass is generated by the cosmological term. We construct a renormalization group (RG) improved effective action by integrating out one loop vacuum fluctuations of the antisymmetric tensor field and show that, in the limit when the RG scale goes to zero, the Hubble parameter - and thus the effective cosmological constant - relaxes rapidly to zero. We thus conclude that quantum loop effects in de Sitter space can dramatically change the infrared sector of the on-shell gravity, making the expansion rate insensitive to the original (bare) cosmological constant.
\end{abstract}

*T.Janssen2@phys.uu.nl

†T.Prokopec@phys.uu.nl 


\section{INTRODUCTION}

In modern quantum field theories, the vacuum is never really empty. A clear example of this is the harmonic oscillator with fundamental frequency $\omega$, whose ground state has an energy $E=\hbar \omega / 2$. This nonzero 'zero point' energy is interpreted as the energy that is always present, even when the oscillator is not excited. One could see this as a manifestation of Heisenberg's uncertainty principle: the oscillator is never completely at rest [1, 2].

The zero point energy of the quantum harmonic oscillator is an example of a very general phenomenon in quantum (field) theories. The quantum vacuum gets enhanced by loop Feynman diagrams. In quantum field theory these diagrams represent a shift in the potential energy and such a shift is, like in classical mechanics, in general unobservable. Therefore often the normal ordering prescription is used, which removes the zero point energy. However, there are special cases, like the Casimir effect, where the vacuum energy does become important. The experimental verification [3] of the Casimir effect [4] however, may not prove the existence of the vacuum energy [5].

\section{A. The cosmological constant problem}

The problems with vacuum energy start to arise as soon as we start to consider general relativity (GR). Whereas in quantum theories, energies are usually observable as the difference between some excitation of the Hamiltonian and the ground state, this is not the case in GR. In GR curvature is sourced by the energy momentum tensor $T_{\mu \nu}$ according to Einstein's equations

$$
R_{\mu \nu}-\frac{1}{2} R g_{\mu \nu}-\Lambda_{g} g_{\mu \nu}=-\frac{1}{2 Q} T_{\mu \nu}
$$

where $R$ and $R_{\mu \nu}$ are the Ricci scalar and tensor respectively, $g_{\mu \nu}$ the metric tensor, $Q$ is related to Newtons constant $G_{N}$ as $Q \equiv\left(16 \pi G_{N}\right)^{-1}$ and $\Lambda_{g}$ is a possible geometrical cosmological constant. $T_{\mu \nu}$ is sourced by any form of energy, including the vacuum energy and in fact Lorentz invariance implies that

$$
T_{\mu \nu}^{\mathrm{vac}}=-\langle\rho\rangle g_{\mu \nu}
$$

where $\langle\rho\rangle$ is the energy density associated with the zero point energy. It is thus clear from (1) that the zero point energy sources gravity in exactly the same way as a positive cosmological constant would. In fact we could write

$$
\Lambda_{\mathrm{c}}=\Lambda_{\rho}+\Lambda_{g}
$$


where $\Lambda_{\mathrm{c}}$ is the total, classical cosmological constant. It is a measurable quantity and current observations [6, 7, 8, 9, 10] indicate that

$$
\Lambda_{\mathrm{c}} \leq 3 H_{0}^{2} \Omega_{\mathrm{DE}} \simeq 5 \times 10^{-84}(\mathrm{GeV})^{2}
$$

where $H_{0} \simeq 1.5 \times 10^{-42} \mathrm{GeV}$ denotes the Hubble parameter today and $\Omega_{\mathrm{DE}} \simeq 0.73$ is the dark energy density in the units of the critical density. However, standard calculations in quantum theory tell us that

$$
\Lambda_{\rho} \sim \frac{M_{X}^{4}}{m_{p}^{2}},
$$

where $m_{p}=1.2 \times 10^{19} \mathrm{GeV}$ is the Planck mass and $M_{X}$ is the maximum mass scale which contributes to the vacuum energy. In the most optimistic case, this could be the QCD scale $(\sim 200 \mathrm{MeV})$, but more realistically it may be as high as $M_{\mathrm{GUT}} \sim 10^{16} \mathrm{GeV}$ or even $m_{p}$. The cosmological constant problem can be formulated as the huge discrepancy between the observed value $\Lambda_{\mathrm{c}}$ and $\Lambda_{\rho}$. Even if $M_{X}$ is at the QCD scale the difference is 40 orders of magnitude, while if $M_{X}$ is at the Planck scale, the difference is an astonishing 122 orders of magnitude. Of course one could tune $\Lambda_{g}$ to fit the observations. This however seems very unnatural, in particular when one takes account of phase transitions in the early Universe during which the vacuum energy is generally changed by an amount typically many orders of magnitude larger than the observed value (4). Many attempts have been made in the past to try to understand the cosmological constant problem [1, 2]. Most of these attempts depend on new physical ideas, like new symmetries or breaking the equivalence principle. However, ordinary quantum field theory has a property that might shed some light on this problem, namely the renormalization group $(\mathrm{RG})$.

\section{B. Renormalization Group}

It is a well established fact [11] [12] that upon regularizing and renormalizing a divergence in quantum field theory, one inevitably introduces a renormalization scale $\mu$. Since this scale is arbitrary, physical observables should be independent of this scale. This requirement is imposed by the renormalization group equations. Solving these equations leads to the RG improved theory, and the coupling constants of the theory, including $\Lambda$, become a function of $\mu$. Since $\Lambda$ now runs with the energy scale $\mu$, one might hope that at cosmological scales $\Lambda$ will run towards zero, independent of its huge value in the ultraviolet where (5) is calculated. Since the cosmological constant problem can be seen as a conflict between scales: the ultraviolet given by $M_{X}$ where $\Lambda_{\rho}$ is defined and the infrared where $\Lambda_{\text {eff }}$ is measured, it seems mandatory to study the RG behavior of $\Lambda$. 
In [13] [14] [15] it is investigated how $\Lambda$ runs with $\mu$, when $\Lambda_{\rho}$ is generated by the Standard Model particles. $\Lambda_{\text {eff }}$ as it is observed is then evaluated at $\mu=H_{0}$, the Hubble parameter today. The result of these calculations is that $\Lambda$ varies only very mildly (usually logarithmically) with $\mu$ and the consensus is that this can never take account of the missing 122 orders of magnitude, though it may make the finetuning issues somewhat less severe.

Another approach is to study the RG behavior of quantum gravity [16, 17, 18]. While this has produced very interesting results (discovery of an ultraviolet fixed point), an explicit calculation of the effective action is still lacking. The main reason for this lies of course in the fact that we do not know what a renormalizable theory of quantum gravity would look like. Another reason is that the authors of [16, 17, 18] in the course of the running project the effective action onto a certain prescribed form (which typically contains $\Lambda, R$ and $R^{2}$ terms but nothing else). This form may be too restrictive since it does not permit e.g. logarithmic dependence on spacetime curvature, which plays an essential role in our one loop investigation.

In this paper we consider in detail the RG behavior of the cosmological constant in a manner that substantially differs from the ideas mentioned above. We explicitly calculate the one loop contributions to the effective action of an antisymmetric tensor field with a mass given by the cosmological constant. Such a field is motivated by nonsymmetric gravitational theories (see section IC), but our results do not depend on its origins. For simplicity our calculations will be done in de Sitter space. Since de Sitter space is essentially empty space, with a cosmological constant, we consider our model exemplary for any space where the energy density is dominated by a cosmological constant.

The Friedmann equation in de Sitter space is given by

$$
H^{2}=\frac{\Lambda_{c}}{3}+\frac{\delta \Lambda_{Q}}{3}
$$

where $\delta \Lambda_{Q}$ stands for quantum loop contributions and therefore depends on the renormalization scale $\mu$. An important observation of this work is that loop corrections, apart from the $\mu$ dependence, can dependent on the Hubble parameter (or more generally on the curvature of space-time) in a rather complex fashion, $\delta \Lambda_{Q}=\delta \Lambda_{Q}\left(\mu, H^{2}\right)$, such that the self-consistent solution for $H^{2}$ in terms of $\Lambda_{\mathrm{c}}$ as specified by Eq. (6) is in general of the form,

$$
H^{2}=\Lambda_{\mathrm{eff}}\left(\Lambda_{\mathrm{c}}, \mu\right) / 3
$$

where $\Lambda_{\text {eff }}$ can be substantially different from $\Lambda_{\mathrm{c}}$ of the original theory. An important question is at what scale $\mu$ one should evaluate the quantum corrections. In other 
words: what is our ultraviolet scale and what is the infrared scale where we measure $\Lambda_{\text {eff }}$ ? It is often argued in the literature that a natural infrared scale for $\mu$ is the Hubble scale. This means that the Hubble length $H^{-1}$ is taken to be the longest relevant wavelength. Causality arguments are sometimes invoked to justify that. The ultraviolet scale is then given by $m_{p}$ or $M_{X}$. We however do not agree with this approach. In accelerating space times modes grow 'super-Hubble' and there is no reason to assume that these modes do not have an effect on the geometry or are unobservable. Therefore a proper treatment of the infrared behavior of the theory must take these modes into account and one should let $\mu$ run down to the energy of the largest super-hubble mode, which is much less then $H$. As for the ultraviolet scale the problem with choosing $m_{p}$ (or $\left.M_{X}\right)$ is that, while $\mu$ runs to the infrared, the ratio $m_{p} / \mu$ becomes huge. This usually spoils the trustability of the one loop expansion. Because of these reasons, we define our ultraviolet theory at $\mu=H$, the Hubble parameter. This makes sense, since not only is the scale $H$ naturally present in the theory, it is also because of the Friedmann equation (6) a natural UV scale. This is because the ultraviolet $\Lambda_{c}$ is huge (see equation (5)). Furthermore, if we decrease $\mu$, we find that also $H$ decreases. Therefore the ratio $H / \mu$ stays closer to one then for any other choice of initial $\mu$, which makes the RG results more trustable.

As a last remark, we point out that a substantial difference between $\Lambda_{\text {eff }}$ and $\Lambda_{c}$ can only be obtained if the quantum radiative effects are comparable to the tree level cosmological constant. While this necessarily makes the loop expansion problematic, we are not aware of any fundamental reason why this compensation should not take place. An example of such a behavior - dubbed attractor mechanism - is considered in Ref. [19], where quantum loop matter fluctuations generate a term which exhibits logarithmic dependence on the Hubble parameter, and which compensates the tree level cosmological term. The important difference between Ref. [19] and the work at hand is that the compensation mechanism in [19] is realised via the dynamics of a scalar field in an effective

potential generated by quantum loop corrections, while here the compensation occurs as a result of sending the RG scale to zero.

\section{Nonsymmetric gravitational theories}

Nonsymmetric gravitational theories (NGTs) are extensions of general relativity (GR), where the standard axiom that the metric is symmetric is dropped [20] [21] [22]. One can therefore make a 
decomposition of the general metric $c_{\mu \nu}$ :

$$
c_{\mu \nu}=g_{\mu \nu}+B_{\mu \nu} \quad g_{\mu \nu} \equiv c_{(\mu \nu)} \quad B_{\mu \nu} \equiv c_{[\mu \nu]},
$$

where $(\cdot)$ and [.] indicate normalized symmetrization and anti-symmetrization, respectively. One of the reasons why such a theory is interesting to study, is that it provides a natural, geometric source for torsion [23]. Another interesting property is that the massive antisymmetric tensor field might act as the dark matter component in the universe [24] [25] [26]. Since GR is a highly successful it makes sense to consider NGT in the limit of a small B-field. In [27] it was shown that the only Lagrangian that does not lead to an unstable field evolution is given by (for the more precise form of this Lagrangian see Eq. (26]) )

$$
\mathcal{L}=\sqrt{-g}\left[Q(R-2 \Lambda)+\frac{b}{144} R^{2}-\frac{1}{12} F^{2}-\frac{1}{4} m^{2} B^{2}\right]+\mathcal{O}\left(B^{3}\right) .
$$

Here the curvature term $R$ refer to the curvature of the GR background and $F_{\mu \nu \rho}$ is the field strength associated with $B_{\mu \nu}$. The mass term is given by

$$
m^{2}=\omega \Lambda+\frac{\chi}{12} R+\frac{\rho}{144 m_{p}^{2}} R^{2}
$$

with $\omega, \chi$ and $\rho$ undetermined constants from the theory. The cosmological constant, as it enters (9), is the sum of the geometric $\left(\Lambda_{g}\right)$ and matter $\left(\Lambda_{\rho}\right)$ contributions. The fact that the mass of the $B$ field is proportional to the cosmological term is extremely interesting, since in principle it induces a large back-reaction on the total energy of the vacuum. This is the question we investigate in detail in this paper.

Most of the conclusions of this paper however are not dependent on the fact that we are dealing with antisymmetric tensor fields. For example a scalar field with a mass proportional to $\Lambda$, as might arise in Kaluza-Klein type theories, would almost certainly lead to similar conclusions.

\section{Overview}

The contents of our paper are as follows. In section II, we discuss some properties of de Sitter space and show how to derive the propagator for the $B$-field. In section III we derive the one loop effective action and in section [V we renormalize the theory and RG improve it. In section $\nabla$ we calculate the Friedmann equation and show how the $B$-field loops alter the effective vacuum energy. We discuss and summarise our results in section VI. 


\section{PROPAGATORS IN DE SITTER SPACE}

In this section we first review basic properties of de Sitter space and then we sketch a standard derivation for the de Sitter invariant scalar propagator. Having done this, we show how to construct the propagator for a massive antisymmetric tensor field in de Sitter space. We show that the propagator can be written as the sum of two scalar propagators with different amounts of conformal coupling. This is similar as in the case of the photon propagator [28, 29] in de Sitter space. In this section we raise and lower indices with $\eta^{\mu \nu}$ and $\eta_{\mu \nu}$, respectively, where $\eta_{\mu \nu}=\operatorname{diag}(-1,1,1, .$.$) is the$ Minkowski metric in D space-time dimensions.

\section{A. de Sitter space}

For pedagogical reasons we begin by introducing four dimensional de Sitter space (later we shall work with general D dimensional de Sitter space), which is the hypersurface given by the equation [30] [19]

$$
-X_{0}^{2}+X_{1}^{2}+X_{2}^{2}+X_{3}^{2}+X_{4}^{2}=\frac{1}{H^{2}}
$$

embedded in 5-dimensional Minkowski space-time, where $H$ is the Hubble parameter. The isometry group of de Sitter space, $S O(1,4)$, is manifest in this embedding. We shall use flat coordinates, which cover only half of the de Sitter manifold, given by $(i=1,2,3)$

$$
\begin{aligned}
X_{0} & =\frac{1}{H} \sinh (H t)+\frac{H}{2} x_{i} x^{i} e^{H t} \\
X_{i} & =e^{H t} x_{i} \\
X_{4} & =\frac{1}{H} \cosh (H t)-\frac{H}{2} x_{i} x^{i} e^{H t}, \\
-\infty & <t, x_{i}<\infty .
\end{aligned}
$$

In these coordinates the metric reads

$$
g_{\mu \nu}=\operatorname{diag}\left(-1, a^{2}, a^{2}, a^{2}\right), \quad a=e^{H t},
$$

which we can write in conformal form by changing coordinates to conformal time $\eta$ defined as $a d \eta=$ $d t$ :

$$
g_{\mu \nu}=\operatorname{diag}\left(-a^{2}, a^{2}, a^{2}, a^{2}\right)=a^{2} \eta_{\mu \nu}, \quad a=-\frac{1}{H \eta}, \quad \eta<0 .
$$

We define the de Sitter invariant distance functions [31] 32]

$$
Z\left(X ; X^{\prime}\right)=H^{2} \sum_{A, B=0}^{4} \eta_{A B} X^{A} X^{B \prime}=1-\frac{1}{2} Y\left(X ; X^{\prime}\right)
$$


In conformal coordinates (12) these functions read

$$
\begin{aligned}
z\left(x ; x^{\prime}\right) & =1-\frac{1}{2} y\left(x ; x^{\prime}\right) \\
y\left(x ; x^{\prime}\right) & =a a^{\prime} H^{2} \Delta x^{2}\left(x ; x^{\prime}\right) \\
\Delta x^{2}\left(x ; x^{\prime}\right) & =-\left(\left|\eta-\eta^{\prime}\right|-i \epsilon\right)^{2}+\left\|\vec{x}-\vec{x}^{\prime}\right\|^{2},
\end{aligned}
$$

where $Y\left(X ; X^{\prime}\right)=y\left(x ; x^{\prime}\right), Z\left(X ; X^{\prime}\right)=z\left(x ; x^{\prime}\right), a=a(\eta)$ and $a^{\prime}=a\left(\eta^{\prime}\right)$ are functions given in (14) and $\epsilon>0$ refers to the Feynman (time ordered) pole prescription. The function $y=y\left(x ; x^{\prime}\right)$ is related to the invariant length $\ell=\ell\left(x ; x^{\prime}\right)$ between points $x$ and $x^{\prime}$ as,

$$
y\left(x ; x^{\prime}\right)=4 \sin ^{2}\left(\frac{1}{2} H \ell\left(x ; x^{\prime}\right)\right) .
$$

\section{B. Scalar propagator in de Sitter space}

The de Sitter invariant scalar propagator for a massive scalar field is the expectation value

$$
i \Delta\left(x ; x^{\prime}\right)=\left\langle x\left|\frac{i}{\sqrt{-g}\left(\square-m^{2}-\xi R_{D}\right)}\right| x^{\prime}\right\rangle,
$$

where $R_{D}$ is the $D$-dimensional Ricci scalar, which in de Sitter space-time is given by $R_{D}=D(D-$ 1) $H^{2}$. $\square$ is the d'Alembertian. The propagator (18) satisfies the following Klein-Gordon equation

$$
\sqrt{-g}\left(\square-m^{2}-\xi R_{D}\right) i \Delta\left(x ; x^{\prime}\right)=i \delta^{D}\left(x-x^{\prime}\right)
$$

where $\delta^{D}$ is the $D$-dimensional Dirac delta. The de Sitter invariant form of (19) is

$$
\left[\left(1-z^{2}\right) \frac{d^{2}}{d z^{2}}-D z \frac{d}{d z}-\frac{m^{2}+\xi R_{D}}{H^{2}}\right] i G(y)=\frac{i \delta^{D}\left(x-x^{\prime}\right)}{a^{D} H^{2}},
$$

where the invariant propagator is defined as $i G(y)=i \Delta\left(x ; x^{\prime}\right)$ and we used

$$
\begin{aligned}
\partial_{\mu} & =\left(\partial_{\mu} z\right) \frac{d}{d z}=-\frac{1}{2} H a\left(\delta_{\mu}^{0} y+2 a^{\prime} H \Delta x_{\mu}\right) \frac{d}{d z} \\
\square & =(\sqrt{-g})^{-1} \partial_{\mu} g^{\mu \nu} \sqrt{-g} \partial_{\nu}=\frac{1}{a^{D}} \partial_{\mu} a^{D-2} \eta^{\mu \nu} \partial_{\nu} \\
& =H^{2}\left(1-z^{2}\right) \frac{d^{2}}{d z^{2}}+\left(a^{-2} \eta^{\mu \nu} \partial_{\mu} \partial_{\nu} z-a^{-1}(D-2) H\left(\partial_{0} z\right)\right) \frac{d}{d z},
\end{aligned}
$$

where $z$ and $y$ are defined in (16) . Eq. (20) is a hypergeometric equation, whose general solution is given in terms of hypergeometric functions

$$
i G(y)=c_{1}{ }_{2} F_{1}\left(\frac{D-1}{2}+\nu, \frac{D-1}{2}-\nu ; \frac{D}{2} ; 1-\frac{y}{4}\right)+c_{2}{ }_{2} F_{1}\left(\frac{D-1}{2}+\nu, \frac{D-1}{2}-\nu ; \frac{D}{2} ; \frac{y}{4}\right),
$$

where

$$
\nu=\left(\frac{(D-1)^{2}}{4}-\frac{m^{2}+\xi R_{D}}{H^{2}}\right)^{1 / 2}
$$


The constants $c_{1}$ and $c_{2}$ are uniquely fixed if we require that near the lightcone the solution reduces to the Hadamard form, while there is no singularity at the antipodal lightcone (which would lead to $\alpha$-vacua [45]). This means we require:

$$
\begin{aligned}
& \lim _{y \rightarrow 0} i G(y)=\frac{H^{D-2}}{(4 \pi)^{D / 2}} \Gamma\left(\frac{D}{2}-1\right) y^{1-\frac{D}{2}} \\
& \lim _{y \rightarrow 4} i G(y)=0
\end{aligned}
$$

which uniquely specifies both constants in Eq. (22) and we arrive at the well known Chernikov-Tagirov scalar propagator in de Sitter space [33]

$$
i G(y)=\frac{H^{D-2}}{(4 \pi)^{D / 2}} \frac{\Gamma\left(\frac{D-1}{2}+\nu\right) \Gamma\left(\frac{D-1}{2}-\nu\right)}{\Gamma\left(\frac{D}{2}\right)}{ }_{2} F_{1}\left(\frac{D-1}{2}+\nu, \frac{D-1}{2}-\nu ; \frac{D}{2} ; 1-\frac{y}{4}\right) .
$$

\section{Calculating the $B$-field propagator}

In this section we calculate the propagator for the anti-symmetric tensor field ( $B$-field) in de Sitter space. Our starting lagrangian is [27]

$$
\mathcal{L}=\sqrt{-g}\left(Q(R-2 \Lambda)+\frac{b}{144} R^{2}-\frac{1}{12} g^{\alpha \mu} g^{\beta \nu} g^{\gamma \rho} F_{\alpha \beta \gamma} F_{\mu \nu \rho}-\frac{1}{4} m^{2} g^{\alpha \mu} g^{\beta \nu} B_{\alpha \beta} B_{\mu \nu}\right),
$$

where $R$ is the Ricci scalar, $g=\operatorname{det}\left[g_{\mu \nu}\right]$ and

$$
\begin{gathered}
m^{2}=\omega \Lambda+\chi H^{2}, \quad Q=\frac{1}{16 \pi G} \\
F_{\alpha \beta \gamma}=\partial_{\alpha} B_{\beta \gamma}+\partial_{\beta} B_{\gamma \alpha}+\partial_{\gamma} B_{\alpha \beta} .
\end{gathered}
$$

Notice that in principle there also is a $H^{4}$ contribution to $\mathrm{m}^{2}$ however there is nothing that forbids us to set this contribution to zero and we do so for simplicity. We will focus on the B-field contribution (remember that we raise/lower indices with $\eta^{\mu \nu} / \eta_{\mu \nu}$ and work in the conformal coordinates of de Sitter space (14))

$$
\begin{aligned}
\mathcal{L}_{B}= & a^{D}\left(-\frac{1}{12} a^{-6} F_{\alpha \beta \gamma} F_{\mu \nu \rho} \eta^{\alpha \mu} \eta^{\beta \nu} \eta^{\gamma \rho}-\frac{1}{4} a^{-4} m^{2} B_{\alpha \beta} B_{\mu \nu} \eta^{\alpha \mu} \eta^{\beta \nu}\right) \\
= & \left.\frac{1}{4} B_{\nu \rho} \partial_{\mu}\left(a^{D-6}\left[\partial_{\alpha} B_{\beta \gamma}+\partial_{\beta} B_{\gamma \alpha}+\partial_{\gamma} B_{\alpha \beta}\right]\right) \eta^{\alpha \mu} \eta^{\beta \nu} \eta^{\gamma \rho}-\frac{1}{4} a^{D-4} m^{2} B_{\alpha \beta} B_{\mu \nu} \eta^{\alpha \mu} \eta^{\beta \nu}\right) \\
= & \frac{1}{4} B^{\beta \gamma} \partial^{\mu}\left(a^{D-6} \partial_{\mu} B_{\beta \gamma}\right)+\frac{1}{2} a^{D-6}\left(\partial^{\alpha} B_{\gamma \alpha}-(D-6) a H B_{\gamma 0}\right)\left(\partial_{\beta} B^{\gamma \beta}-(D-6) a H B^{0 \gamma}\right) \\
& \quad+\frac{1}{2}(D-6) H^{2} a^{D-4} B_{\gamma 0} B^{0 \gamma}-\frac{1}{4} a^{D-4} m^{2} B_{\alpha \beta} B_{\mu \nu} \eta^{\alpha \mu} \eta^{\beta \nu},
\end{aligned}
$$

where in the second line we have dropped a total derivative. Next we add a gauge fixing term [46]:

$$
\mathcal{L}_{G F}=-\frac{1}{2} a^{D-6}\left(\partial^{\alpha} B_{\gamma \alpha}-(D-6) a H B_{\gamma 0}\right)\left(\partial_{\beta} B^{\gamma \beta}-(D-6) a H B^{0 \gamma}\right)
$$


to obtain

$$
\begin{aligned}
\mathcal{L}_{B-G F} & =\frac{1}{4}\left(B^{\beta \gamma} \partial^{\alpha}\left(a^{D-6} \partial_{\alpha}\right) B_{\beta \gamma}-a^{D-4} m^{2} B^{\beta \gamma} B_{\beta \gamma}-2(D-6) H^{2} a^{D-4} B_{\gamma 0} B^{\gamma 0}\right) \\
& =-\frac{1}{2} B^{\mu \nu} \mathcal{D}_{\mu \nu}^{\rho \sigma} B_{\rho \sigma},
\end{aligned}
$$

where we have defined

$$
\mathcal{D}_{\mu \nu}^{\rho \sigma}=-\frac{1}{2}\left[\left(\partial^{\alpha} a^{D-6} \partial_{\alpha}-a^{D-4} m^{2}\right) \delta_{\mu}^{[\rho} \delta_{\nu}^{\sigma]}-2(D-6) H^{2} a^{D-4} \delta_{[\nu}^{0} \bar{\delta}_{\mu]}^{[\rho} \delta_{0}^{\sigma]}\right]
$$

and

$$
\bar{\delta}_{\nu}^{\mu}=\delta_{\nu}^{\mu}-\delta_{0}^{\mu} \delta_{\nu}^{0}, \quad \bar{\eta}_{\mu \nu}=\eta_{\mu \alpha} \bar{\delta}_{\nu}^{\alpha}=\eta_{\mu \nu}+\delta_{\mu}^{0} \delta_{\nu}^{0}
$$

The propagator is given by

$$
i \mathcal{D}_{\mu \nu}^{\rho \sigma}\left[\rho \sigma \Delta_{\alpha \beta}\right]\left(x ; x^{\prime}\right)=i \eta_{\alpha[\mu} \eta_{\nu] \beta} \delta^{D}\left(x-x^{\prime}\right)
$$

We use the following ansatz for the propagator (the subscripts 2 and 3 will become clear shortly)

$$
\left.{ }_{\rho \sigma} \Delta_{\alpha \beta}\left(x ; x^{\prime}\right)=-2 a^{2} a^{\prime 2}\left[\bar{\eta}_{\alpha[\rho} \bar{\eta}_{\sigma] \beta} \Delta_{2}\left(x ; x^{\prime}\right)+\delta_{[\sigma}^{0} \bar{\eta}_{\rho][\alpha} \delta_{\beta}^{0}\right] \Delta_{3}\left(x ; x^{\prime}\right)\right]
$$

With the help of the identity

$$
\begin{aligned}
\left(\partial^{\alpha} a^{D-6} \partial_{\alpha}\right)\left(2 a^{2} a^{\prime 2} \Delta\right) & =a^{D}\left(\partial^{\sigma} a^{D-2} \partial_{\sigma}-2(D-3) H^{2}\right) \Delta \\
& =a^{D}\left(\square-2(D-3) H^{2}\right) \Delta
\end{aligned}
$$

we find that

$$
\begin{aligned}
& \left(\square-2(D-3) H^{2}-m^{2}\right) i \Delta_{2}\left(x ; x^{\prime}\right)=a^{-D} i \delta^{D}\left(x-x^{\prime}\right) \\
& \left(\square-3(D-4) H^{2}-m^{2}\right) i \Delta_{3}\left(x ; x^{\prime}\right)=a^{-D} i \delta^{D}\left(x-x^{\prime}\right) .
\end{aligned}
$$

So we see that, similarly to the case of the photon propagator in [28], that we can write the $B$-field propagator in terms of scalar propagators with various types of coupling to the Ricci scalar.

Our $\Delta_{2}$ corresponds to $\Delta_{C}$ of [28] [34]. In fact the propagators $A, B$ and $C$ of these references correspond to $n=0,1,2$ respectively of

$$
\left(\square-n(D-n-1) H^{2}-m^{2}\right) i \Delta_{n}\left(x ; x^{\prime}\right)=a^{-D} i \delta^{D}\left(x-x^{\prime}\right) .
$$

This equation is solved by (25), with

$$
\begin{aligned}
\nu & \rightarrow \nu_{n}=\left(\frac{(D-2 n-1)^{2}}{4}-\frac{m^{2}}{H^{2}}\right)^{1 / 2} \\
i G(y) & \rightarrow i G_{n}(y) .
\end{aligned}
$$




\section{THE EFFECTIVE ACTION}

Since the $B$-field appears quadratically in our lagrangian (26), it can be integrated out. Thus we get (up to an irrelevant normalization constant)

$$
\exp \left[i \Gamma\left(g_{\mu \nu}\right)\right]=\int \mathcal{D} B \exp \left[i S\left(g_{\mu \nu}, B_{\mu \nu}\right)\right]=\exp \left[i S_{H E}\left(g_{\mu \nu}\right)\right] \frac{1}{\sqrt{\operatorname{Det}\left[D^{\mu \nu \rho \sigma}\right]}}
$$

and therefore

$$
\Gamma\left(g_{\mu \nu}\right)=S_{H E}\left(g_{\mu \nu}\right)+\frac{i}{2} \operatorname{Tr} \ln \left(\mathcal{D}^{\mu \nu \rho \sigma}\right)+\text { higher loops, }
$$

where the trace refers to the spacetime integration $\int d^{D} x \equiv \int_{x}$ and the contraction over the Lorentz indices. Using (31) we find

$$
\begin{aligned}
\Gamma_{B} & \equiv \frac{i}{2} \operatorname{Tr} \ln \left(D^{\mu \nu \rho \sigma}\right) \\
& =\frac{i}{2} \int_{x}\left(\eta_{\rho[\mu} \eta_{\nu] \sigma} \ln \left[-\frac{1}{2}\left(\left(\partial^{\alpha} a^{D-6} \partial_{\alpha}-a^{D-4} m^{2}\right) \eta^{\mu[\rho} \eta^{\sigma] \nu}-2(D-6) H^{2} a^{D-4} \delta_{0}^{[\nu} \bar{\eta}^{\mu][\rho} \delta_{0}^{\sigma]}\right)\right]\right) .
\end{aligned}
$$

The standard technique [12] [35] to get rid of the log is to take a derivative with respect to the mass and then write $\Gamma$ in terms of the propagator. In our case however, we have to take a log of two expressions which are, because of the tensorial structure, orthogonal. To take this properly into account we need to take both the derivative with respect to the mass and with respect to $H^{2}$. We obtain

$$
\begin{aligned}
\Gamma_{B}=\frac{i}{2} \int_{x}\left[\int d m^{2}\left(\eta_{\rho[\mu} \eta_{\nu] \sigma}{ }^{\rho \sigma} \Delta^{\alpha \beta}\right](x ; x)\left(\frac{1}{2} a^{D-4}\right) \delta_{\alpha}^{[\mu} \delta_{\beta}^{\nu]}\right) \\
\left.\left.-\quad \int d H^{2}\left(\eta_{\rho[\mu} \eta_{\nu] \sigma}{ }^{\rho \sigma} \Delta^{\alpha \beta}\right](x ; x)\left((D-6) a^{D-4} \delta_{0}^{[\nu} \eta_{[\alpha}^{\mu]} \delta_{\beta]}^{0}\right)\right)\right] \\
=-\frac{1}{4} \int_{x}\left[\int d m^{2} a^{D}\left((D-1)(D-2) i \Delta_{2}(x ; x)-(D-1) i \Delta_{3}(x ; x)\right)\right. \\
\left.\quad+\int d H^{2} a^{D}(D-1)(D-6) i \Delta_{3}(x ; x)\right] \\
=-\frac{1}{4} \int_{x} a^{D}\left[\int d w(D-1) H^{2}\left((D-2) i \Delta_{2}(x ; x)-i \Delta_{3}(x ; x)\right)\right. \\
\left.\quad+\int d H^{2}(D-1)\left((D-2) w i \Delta_{2}(x ; x)+((D-6)-w) i \Delta_{3}(x ; x)\right)\right] \\
\equiv \Gamma_{w}+\Gamma_{H},
\end{aligned}
$$

where

$$
w \equiv \frac{m^{2}}{H^{2}}
$$


Since at the coincidence limit only the $y^{0}$ term of the propagators contribute (the other powers are $D$-dependent powers and do not contribute in dimensional regularization), we use the following expression for the scalar propagators.

$$
\left.i \Delta_{n}(x ; x)\right|_{y^{0}-\text { term }}=\frac{H^{D-2}}{(4 \pi)^{D / 2}} \Gamma\left(1-\frac{D}{2}\right) \frac{\Gamma\left(\frac{D-1}{2}+\nu_{n}\right) \Gamma\left(\frac{D-1}{2}-\nu_{n}\right)}{\Gamma\left(\frac{1}{2}+\nu_{n}\right) \Gamma\left(\frac{1}{2}-\nu_{n}\right)},
$$

with $\nu_{n}$ defined in (38). In order to renormalize the theory, we need to split the effective action (42), with propagators given by (44), in its finite and infinite parts. This separation is done in Appendix A. The final result is

$$
\begin{aligned}
\Gamma_{B}= & -\int_{x} \sqrt{-g}[ \\
& \frac{D-1}{4(4 \pi)^{D / 2}} \Gamma(1-D / 2)\left(H^{4} \frac{1}{2 D}\left[8(D-5)(D-6)+2\left(24+D\left(D^{2}-6 D+4\right)\right) \chi+(D-3)(D+4) \chi^{2}\right]\right. \\
& \left.\quad+H^{2} \omega \Lambda\left(4+D^{2}+D(\chi-6)-\frac{12(\chi-2)}{D}+\chi\right)+(\omega \Lambda)^{2} \frac{(D-3)(D+4)}{2 D}\right) \mu^{D-4} \\
& +\frac{3}{64 \pi^{2}}\left(\left((\omega \Lambda)^{2}+2(1+\chi) \omega \Lambda H^{2}+2\left(1+\chi+\frac{\chi^{2}}{2}\right) H^{4}\right) \ln \left(\frac{\omega \Lambda+\chi H^{2}}{\mu^{2}}\right)-\frac{31}{15} H^{4} \ln \left(\frac{\omega \Lambda+\chi H^{2}}{H^{2}}\right)\right. \\
& \left.\left.\quad-\frac{(\omega \Lambda)^{2}}{4}+\frac{H^{2} \omega \Lambda}{2}(1-\chi)+\frac{\chi H^{4}}{2}\left(1-\frac{\chi}{2}\right)+\frac{13}{10} H^{4}\right)\right]+\mathcal{O}\left(H^{4} \frac{H^{2}}{m^{2}}\right) \\
\equiv & \int_{x} \delta \mathcal{L} .
\end{aligned}
$$

In this calculation we used the approximation that

$$
\frac{H^{2}}{\omega \Lambda+\chi H^{2}} \ll 1
$$

which we justify in section $\mathrm{VB} 2$.

The effective action(45) can be simplified further in the special case when $\chi \leq \mathcal{O}(1)$, since then our approximation (46) is equivalent to $H^{2} /(\omega \Lambda) \ll 1$. In this case we should replace the logs in (45):

$$
\begin{aligned}
& \ln \left(\frac{\omega \Lambda+\chi H^{2}}{H^{2}}\right) \rightarrow \ln \left(\frac{\omega \Lambda}{H^{2}}\right)+\frac{\chi H^{2}}{\omega \Lambda}-\frac{1}{2}\left(\frac{\chi H^{2}}{\omega \Lambda}\right)^{2} \\
& \ln \left(\frac{\omega \Lambda+\chi H^{2}}{\mu^{2}}\right) \rightarrow \ln \left(\frac{\omega \Lambda}{\mu^{2}}\right)+\frac{\chi H^{2}}{\omega \Lambda}-\frac{1}{2}\left(\frac{\chi H^{2}}{\omega \Lambda}\right)^{2} .
\end{aligned}
$$

\section{RENORMALIZATION}

The Lagrangian we wish to renormalize is (remember that in de Sitter space $R=12 H^{2}$ )

$$
\mathcal{L}=\sqrt{-g}\left(Q\left(12 H^{2}-2 \Lambda\right)+b H^{4}+\delta \mathcal{L}\right)
$$


and to do so we add the following counterterms

$$
\mathcal{L}_{C T}=\sqrt{-g}\left(\Lambda_{c}+Q_{c} H^{2}+b_{c} H^{4}\right) .
$$

We use a regularization scheme in which the counterterms remove all non-log terms from $\delta \mathcal{L}$. This scheme has the advantage that the equations of motion become relatively simple. The results in other regularization schemes are related to this regularization scheme by a finite shift in the coupling constants. A potential disadvantage of this scheme is that it is not immediately clear what are the physical Newton and cosmological constant. This issue we shall study in more detail in section IVB. Using our regularization scheme the lagrangian becomes

$$
\begin{aligned}
\mathcal{L}_{\text {ren }}=\sqrt{-g}( & Q\left(12 H^{2}-2 \Lambda\right)+b H^{4} \\
& -\frac{3}{64 \pi^{2}}\left[\left((\omega \Lambda)^{2}+2(1+\chi) \omega \Lambda H^{2}+2\left(1+\chi+\chi^{2} / 2\right) H^{4}\right) \ln \left(\frac{\omega \Lambda+\chi H^{2}}{\mu^{2}}\right)\right. \\
& \left.\left.-\frac{31}{15} H^{4} \ln \left(\frac{\omega \Lambda+\chi H^{2}}{H^{2}}\right)\right]+\mathcal{O}\left(\frac{H^{6}}{m^{2}}\right)\right) .
\end{aligned}
$$

Notice that if $\chi \sim \mathcal{O}(1)$, Eq. (46) implies that the replacement (47) is applicable, one should only replace the logs, since the other terms generated by (477) are removed in our regularization scheme.

\section{A. RG improvement}

The renormalized lagrangian (50) still contains the arbitrary mass-scale $\mu$. The dependence on this scale is unphysical, since it is introduced by the counterterms. Removing the $\mu$ dependence is tantamount to the renormalization group improvement [36] [37] [12] [11]. From

$$
\mu \partial_{\mu} \mathcal{L}_{\mathrm{ren}}=\sqrt{-g} \frac{3}{32 \pi^{2}}\left((\omega \Lambda)^{2}+2(1+\chi) \omega \Lambda H^{2}+2\left(1+\chi+\frac{1}{2} \chi^{2}\right) H^{4}\right)
$$

we read off the beta-functions

$$
\begin{aligned}
& \beta_{\Lambda}=\frac{3}{64 \pi^{2}} \frac{(\omega \Lambda)^{2}}{Q}+\frac{1+\chi}{64 \pi^{2}} \frac{\omega \Lambda^{2}}{Q} \\
& \beta_{Q}=-\frac{1+\chi}{64 \pi^{2}} \omega \Lambda \\
& \beta_{b}=-\frac{3}{16 \pi^{2}}\left(1+\chi+\frac{1}{2} \chi^{2}\right) .
\end{aligned}
$$

The idea is now to 'improve' our lagrangian by imposing the renormalization group equation, which in our case (where wave function renormalization can be neglected) can be well approximated by the Callan-Symanzik equation

$$
\left(\mu \partial_{\mu}+\beta_{\Lambda} \partial_{\Lambda}+\beta_{Q} \partial_{Q}+\beta_{b} \partial_{b}\right) \mathcal{L}_{\text {ren }}=0
$$


We solve this equation by the method of characteristics [37] [38] (see also Appendix [B), which means that we need to make the substitution

$$
\mathcal{L}_{\text {ren }}(\Lambda, Q, b, \mu) \rightarrow \mathcal{L}_{\text {ren }}(\Lambda[t], Q[t], b[t], \mu[t])
$$

where $\Lambda[t], Q[t]$ and $b[t]$ are the solutions of the differential equations

$$
\frac{d}{d t} \Lambda[t]=\beta_{\Lambda}, \quad \frac{d}{d t} Q[t]=\beta_{Q}, \quad \frac{d}{d t} b[t]=\beta_{b}
$$

and

$$
\mu[t]=\mu e^{t}
$$

$t$ is a parameter, independent of the couplings $\Lambda, Q$ or $b$, that we choose to be

$$
t=\ln \left(\frac{H}{\mu}\right)
$$

such that

$$
\mu[t]=H
$$

This choice of $t$ is however not unique, and different choices lead in principle to different RG improved effective actions with different boundary conditions. However, choosing a different $t$ differs from the effective action (61) only at higher order in the coupling constants, and hence we believe that the results presented in this work are generic. On the other hand, since in this work tree level and one loop contributions are comparable, this question does deserve further study.

Our motivation for the choice (57) is the following: first of all the scale $H$ is naturally present in the theory. Moreover, since $\Lambda$ in the ultraviolet is huge (see (5) ), $t=0(\mu=H)$ gives a natural ultraviolet scale. One could argue that $\mu=M_{X}$ or $\mu=m_{p}$ are also natural ultraviolet scales. While this in principle is true, such a choice presents the problem that when $\mu$ runs to zero, $t$ blows up. This is problematic, since this means that the running parameters hit the Landau pole (see (59)). Our choice (57) on the other hand does not present this problem, since we will show that $H$ decreases when $\mu$ decreases in such a way that $t$ never blows up. Thus the choice (57) has the advantage that it results in trustable running from the far ultraviolet to the far infrared.

The solutions to the renormalization group equations 55 are given by

$$
\begin{aligned}
Q[t] & =Q_{0}\left(1-2(2(1+\chi)+3 \omega) \tilde{Q}_{0}^{-1} \omega \Lambda_{0} t\right)^{\frac{1+\chi}{2(1+\chi)+3 \omega}} \\
\Lambda[t] & =\Lambda_{0}\left(1-2(2(1+\chi)+3 \omega) \tilde{Q}_{0}^{-1} \omega \Lambda_{0} t\right)^{-\frac{1+\chi+3 \omega}{2(1+\chi)+3 \omega}} \\
b[t] & =b_{0}-\frac{3}{16 \pi^{2}}\left(1+\chi+\frac{1}{2} \chi^{2}\right) t
\end{aligned}
$$


where a subscript zero means evaluation of the parameter at $t=0$, and

$$
\tilde{Q}_{0}=128 \pi^{2} Q_{0}=8 \pi m_{p}^{2}
$$

where $m_{p}=1.2 \times 10^{19} \mathrm{GeV}$ is the Planck scale.

With the replacement (54) we get the final RG improved lagrangian

$$
\begin{aligned}
\mathcal{L}_{R G} & =\sqrt{-g}\left[Q[t](R-2 \Lambda[t])+b[t] H^{4}\right. \\
& \left.-\frac{3}{64 \pi^{2}}\left((\omega \Lambda[t])^{2}+2(1+\chi) \omega \Lambda[t] H^{2}+2\left(-\frac{1}{30}+\chi+\frac{1}{2} \chi^{2}\right) H^{4}\right) \ln \left(\frac{\omega \Lambda[t]+\chi H^{2}}{H^{2}}\right)\right]+\mathcal{O}\left(\frac{H^{6}}{m^{2}}\right) .
\end{aligned}
$$

This effective lagrangian is finite and independent on the scale $\mu$ (in the sense of the Callan-Symanzik equation), and we use it in the following to study how the one-loop $B$ field quantum corrections influence the Friedmann equation in de Sitter space.

\section{B. Physical parameters and boundary conditions}

Since $H$ appears in the logarithm of (61), it is a priori not clear what we mean with our physical parameters. Since we - naturally - do not want our model to spoil e.g. solar system measurements, it is important to identify physical parameters. We define the physical constants (denoted by subscript p) in a standard way

$$
\begin{aligned}
\Lambda[t]_{p} Q[t]_{p} & \equiv-\frac{1}{2} \lim _{H \rightarrow 0} \frac{\mathcal{L}}{\sqrt{-g}} \\
Q[t]_{p} & \equiv \frac{1}{12} \lim _{H \rightarrow 0} \frac{\partial}{\partial H^{2}} \frac{\mathcal{L}}{\sqrt{-g}} \\
b[t]_{p} & \equiv \frac{1}{2} \lim _{H \rightarrow 0}\left(\frac{\partial}{\partial H^{2}}\right)^{2} \frac{\mathcal{L}}{\sqrt{-g}} .
\end{aligned}
$$

Our analysis in section $\nabla$ shows that $\Lambda_{p}[t]$ is not the relevant parameter that determines the expansion rate of the universe. Instead, the relevant quantity which determines the expansion rate is given by $\Lambda_{\text {eff }}$, which is the self consistent solution to the quantum Friedmann equation.

In the following we assume $\omega \gg \chi+1$ and $\chi \leq \mathcal{O}(1)$, such that we can apply (47) (see also the note after equation (50)). In this limit, the RG improved parameters (59) become

$$
\begin{aligned}
Q[t] & \simeq Q_{0} \\
\Lambda[t] & \simeq \frac{\Lambda_{0}}{1-6 \omega^{2}\left(\tilde{Q}_{0}\right)^{-1} \Lambda_{0} t} \\
b[t] & =b_{0}-\frac{3}{16 \pi^{2}}\left(1+\chi+\frac{1}{2} \chi^{2}\right) t .
\end{aligned}
$$


With these parameters, we calculate (62) for the lagrangian (61) and obtain

$$
\begin{aligned}
Q_{p}[t] \Lambda_{p}[t] & =\lim _{H \rightarrow 0}\left[Q[t] \Lambda[t]+\frac{3}{128 \pi^{2}} \omega^{2} \Lambda[t]^{2} \ln \left(\frac{\omega \Lambda[t]}{H^{2}}\right)\right] \\
& =2 Q_{0} \Lambda[t]+\mathcal{O}\left(\frac{1}{t^{2}}, H^{2}\right) .
\end{aligned}
$$

To calculate the first derivative of $\mathcal{L}_{\mathrm{RG}} / \sqrt{-g}$, first note that the derivative of the parameters $Q[t]$, $\Lambda[t]$ and $b[t]$ can be easily obtained by noting, $\partial_{t}=-\mu \partial_{\mu}$, which is evaluated in Eq. (51), whereby the potentially divergent $1 / H^{2}$ terms cancel out. The result is,

$$
\begin{aligned}
Q_{p}[t] & =Q[t]-\frac{\omega \Lambda[t]}{128 \pi^{2}}\left[(1+\chi) \ln \left(\frac{\omega \Lambda[t]}{H^{2}}\right)+\frac{\chi}{2}\right]+\mathcal{O}\left(\frac{1}{t}, H^{2}\right) \\
& =Q_{0}\left[1-\frac{1+\chi}{3 \omega}\right]+\mathcal{O}\left(\frac{1}{t}, H^{2}\right)
\end{aligned}
$$

where we used

$$
\ln \left(\frac{\omega \Lambda[t]}{H^{2}}\right)=\ln \left(\frac{\omega \Lambda[t]}{\mu^{2}}\right)-2 t \sim-2 t
$$

Equation (65) implies that $Q_{p}$ is equal to $Q_{0}$ plus a small, $t$-independent, correction (remember that we assumed $\omega \gg \chi+1)$. Since this means that the Newton constant does not run with the scale $\mu$, standard gravitational tests are not affected in this limit.

Furthermore we find that, since $Q_{p} \simeq Q_{0}$, Eq. (64) implies that

$$
\Lambda_{p}[t] \simeq 2 \Lambda[t]
$$

and therefore it makes sense to put (see Eq. (15) )

$$
\Lambda_{0}=M_{X}^{4} / m_{p}^{2}
$$

Unfortunately it does not seem to be possible to make a similar statement for $b$. However this is of no great concern to us, since the term $b_{0} H^{4}$ does not contribute to the Friedmann equation and thus it is not expected to influence gravitational tests.

\section{THE FRIEDMANN EQUATION}

From (59) it is clear that $\Lambda$ runs only logarithmically with $\mu$ and this is never sufficient to get $\Lambda[\mu] \rightarrow 0$ in a satisfying manner [2]. The physically relevant quantity however is the effective cosmological constant given by the (modified) Friedmann equation

$$
H^{2}=\frac{\Lambda_{\mathrm{eff}}}{3}
$$


where $\Lambda_{\text {eff }}$ may be very different from $\Lambda$. To get the Friedmann equation we need to calculate

$$
\frac{\partial \mathcal{L}_{\mathrm{RG}}}{\partial g^{\mu \nu}}=0 \rightarrow-\frac{1}{2} \mathcal{L}_{\mathrm{RG}} / \sqrt{-g}+\frac{1}{4} H^{2} \frac{\partial\left(\mathcal{L}_{\mathrm{RG}} / \sqrt{-g}\right)}{\partial H^{2}}+\frac{1}{8} \frac{\partial\left(\mathcal{L}_{\mathrm{RG}} / \sqrt{-g}\right)}{\partial t}=0
$$

for the RG improved lagrangian (61). After some algebra and dividing the result by $-3 Q$ we obtain

$$
\begin{aligned}
& H^{2}\left\{1+(1+\chi) \frac{\omega \Lambda[t]}{\tilde{Q}[t]}-\left(1-(1+\chi+3 \omega) \frac{\omega \Lambda[t]}{\tilde{Q}[t]}\right) \frac{\omega \Lambda[t]}{\tilde{Q}[t]}\left[(1+\chi) \ln \left(\frac{\omega \Lambda[t]+\chi H^{2}}{H^{2}}\right)+1+\frac{\chi}{2}\right]\right\} \\
& -\frac{\Lambda[t]}{3}\left\{1-\frac{3}{2} \frac{\omega^{2} \Lambda[t]}{\tilde{Q}[t]}+\left(1-(1+\chi+3 \omega) \frac{\omega \Lambda[t]}{\tilde{Q}[t]}\right) \frac{3 \omega^{2} \Lambda[t]}{\tilde{Q}[t]}\left[\ln \left(\frac{\omega \Lambda[t]+\chi H^{2}}{H^{2}}\right)+\frac{1}{2}\right]\right\} \\
& +\frac{H^{4}}{\tilde{Q}[t]}\left\{1+\chi+\frac{1}{2} \chi^{2}+\frac{1}{3}\left(1-(1+\chi+3 \omega) \frac{\omega^{2} \Lambda[t]}{\tilde{Q}[t]}\right)\right\}=0,
\end{aligned}
$$

where we have removed a $H^{4} / m^{2}$ term, in agreement with our approximation (46). In order to find the effective cosmological constant, we need to find the self consistent solution of (71) for $H^{2}$.

\section{A. Case 1: $\omega^{2} \Lambda[t] / \tilde{Q}[t] \ll 1$}

At first instance, this case might appear to be the most interesting to look at. In this limit the Friedmann equation reads

$$
H^{2}-\frac{\Lambda[t]}{3}+\alpha \frac{H^{4}}{\tilde{Q}[t]}=0 \quad \alpha \equiv \frac{31}{30}+\chi+\frac{1}{2} \chi^{2},
$$

which is solved by

$$
H^{2}=\tilde{Q}[t] \frac{\sqrt{1+\alpha \frac{4 \Lambda[t]}{3 \tilde{Q}[t]}}-1}{2 \alpha} .
$$

Clearly there is no way in which (73) would lead to $\Lambda_{\text {eff }} \rightarrow 0$. To compare (73) with the standard form of the Friedmann equation, we assume $\alpha \Lambda[t] \ll \tilde{Q}[t]$ and get

$$
H^{2}=\frac{\Lambda[t]}{3}\left[1-\frac{\alpha \Lambda[t]}{3 \tilde{Q}[t]}+\mathcal{O}\left(\frac{\alpha \Lambda[t]}{3 \tilde{Q}[t]}\right)^{2}\right]
$$

Therefore we see that we get a small correction to the Friedmann equation.

On the other hand, one could assume that $\alpha \Lambda[t] \gg \tilde{Q}[t]$. In this case Eq. (73))implies that $H^{2} \simeq$ $\sqrt{\tilde{Q}[t] \Lambda[t] /(3 \alpha)}$, such that $H^{2}$ is given by the geometric mean between $\Lambda[t]$ and the Planck scale $\tilde{Q}_{0}=8 \pi m_{p}^{2}$. This clearly does not help us in addressing the cosmological constant problem. Moreover, since in this limit perturbative calculations are expected to break down, this result is not to be taken too seriously. 


\section{B. Case 2: $\omega \Lambda / \tilde{Q} \ll 1$ and $\omega^{2} \Lambda / \tilde{Q} \sim 1$}

This limit requires that $M_{X}$ is considerably smaller then $m_{p}$ and $\omega$ needs at least to be order 10 . However these assumptions are not unrealistic. For simplicity we also assume that $\omega \gg \chi+1$, so we can use the results of section IVB. Furthermore we assume that $|\chi| \leq \mathcal{O}(1)$, so we can use the approximation (47) (see also the comment following equation (50)). Finally we shall drop the $H^{4} / \tilde{Q}$ terms in the Friedmann equation, since we will be interested in the regime where $H$ becomes small. We now summarize all of our assumptions,

$$
H^{4} / \tilde{Q} \ll H^{2} \ll \omega \Lambda \ll \tilde{Q}, \quad \omega^{2} \Lambda \sim \tilde{Q}, \quad \omega \gg \chi+1 \quad|\chi| \leq 1
$$

With these assumptions the Friedmann equation (71) becomes

$$
H^{2}=\frac{\lambda[t] \tilde{Q}_{0}}{3 \omega^{2}}\left[1+(1-3 \lambda) 3 \lambda L-\frac{9}{2} \lambda^{2}\right]
$$

where we have defined

$$
\begin{aligned}
\lambda & \equiv \frac{\omega^{2} \Lambda[t]}{\tilde{Q}_{0}}=\frac{\lambda_{0}}{1-6 \lambda_{0} t} \\
\lambda_{0} & \equiv \frac{\omega^{2} \Lambda_{0}}{\tilde{Q}_{0}} \simeq \frac{\omega^{2}}{8 \pi} \frac{M_{X}^{4}}{m_{p}^{4}} \\
L & \equiv \ln \left(\frac{\tilde{Q}_{0} \lambda}{\omega H^{2}}\right) .
\end{aligned}
$$

In the second line of (77) we have used the results of section IVB.

If we start the RG flow at $\mu=H$ and let $\mu$ go to zero (see section $(\mathrm{IB}$ ), there are two possibilities. If $H$ decreases slower then $\mu, \lambda[t]$ increases; otherwise $\lambda[t]$ decreases. Furthermore it is clear that if $\lambda$ increases, and $H$ decreases, clearly $L$ will increase. We solve the equation $[$ r.h.s. of (176) $]=0$ in this limit and get (apart from the trivial solution)

$$
\lambda=\frac{1}{3}+\frac{1}{6} \frac{1}{L}-\frac{1}{4} \frac{1}{L^{2}}+\mathcal{O}\left(\frac{1}{L^{3}}\right.
$$

Therefore we see that when $H$ goes to zero, $\lambda$ approaches $1 / 3$ from above. But this means that $\lambda$ ought to decrease. Fortunately this is exactly what happens: for $\lambda<\lambda_{\text {crit }}$ [47], the ratio $H / \mu$ grows and thus $\lambda$ increases. However when $\lambda$ equals $\lambda_{\text {crit }}, H$ starts to decrease faster then $\mu$, so from that point onwards, $\lambda$ actually starts to increase, driving $H^{2}$ towards zero. Before studying this process in more detail in section VB1, let us first determine how fast $H^{2}$ goes to zero as $\lambda \searrow 1 / 3$. To do this, we rewrite the Friedmann equations (176) as follows:

$$
H^{2}=\frac{\tilde{Q}_{0} \lambda}{\omega} \exp \left[\frac{1}{(1-3 \lambda) 3 \lambda}\left(1-\frac{9}{2} \lambda^{2}-\frac{3 \omega^{2} H^{2}}{\tilde{Q}_{0} \lambda}\right)\right]
$$




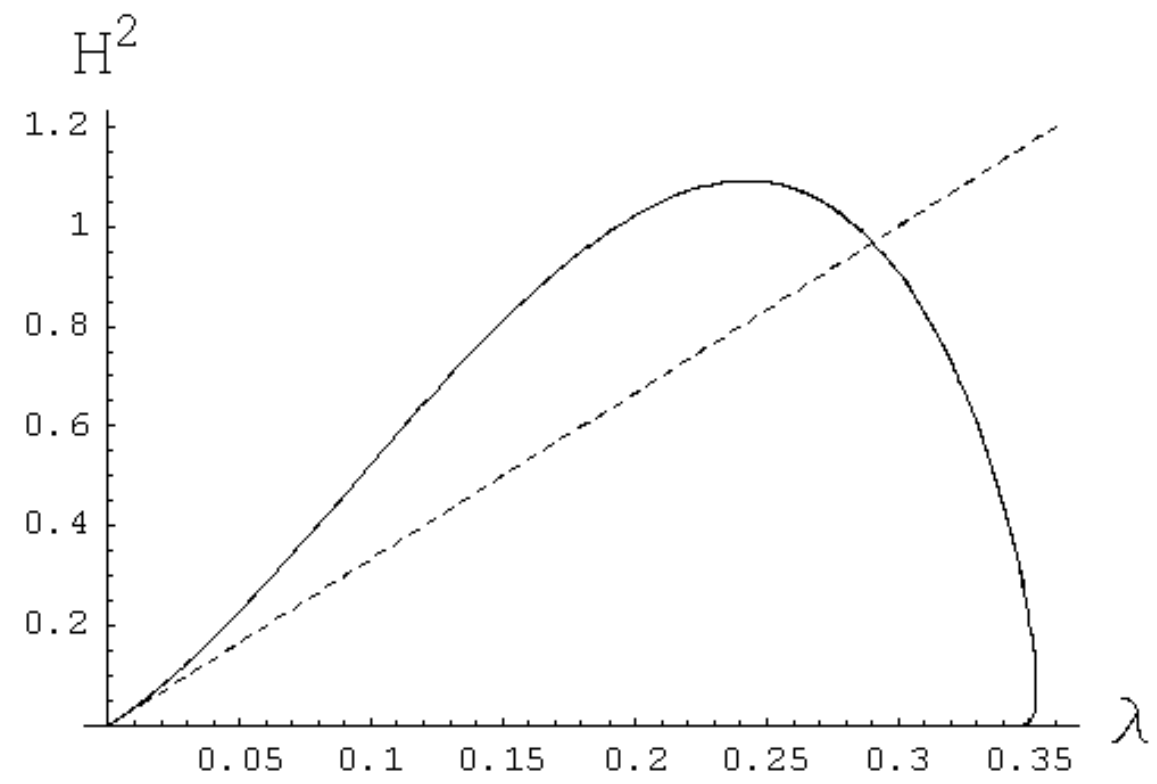

FIG. 1: $H^{2}$ versus $\lambda[t]$. The solid curve is the solution to the Friedmann equation, the dashed line is given by $H^{2}=\Lambda[t] / 3$.

The limit $H \rightarrow 0$ and $\lambda \searrow 1 / 3$ from above thus means approximately:

$$
H^{2} \simeq \frac{\tilde{Q}_{0}}{3 \omega} \exp \left[-\frac{1}{6(\lambda-1 / 3)}\right]
$$

From this equation we see that, since $\lambda$ depends on $\ln (H / \mu)$, we actually have approximately that $H \propto \mu$ and thus we get power-law running instead of logarithmic running. In fact $H$ goes even faster to zero, since we require that $H$ goes to zero faster then $\mu$ and therefore $\lambda$ 'accelerates' towards $1 / 3$.

\section{Numerical analysis of the model in the infrared limit $\mu \rightarrow 0$}

In this section we demonstrate that the behavior described in the previous section, is actually realized in this model. Unfortunately we are unable to solve the Friedmann equation analytically for $H$, therefore we need to rely on numerical analysis. For definiteness we choose $\tilde{Q}_{0}=1000$ and $\omega=10$, however none of the qualitative features of the model depends on these numbers. One does not need to specify $\Lambda_{0}$, since $\Lambda_{0}$ will only define the 'starting point' on the curve (VB 1). While we choose two parameters, we actually have a one parameter family of curves, since the $\tilde{Q}_{0}$ dependence in (76) $)$ can be removed by defining $h^{2} \equiv H^{2} / \tilde{Q}_{0}$.

We solve the Friedmann equation numerically and plot $H^{2}$ versus $\lambda[t]$ (figures VB 1 and 2). The first thing we need to consider is whether, if we start with $0<\lambda_{0}<1 / 3$ and let $\mu$ decrease, $\lambda[t]$ increases 


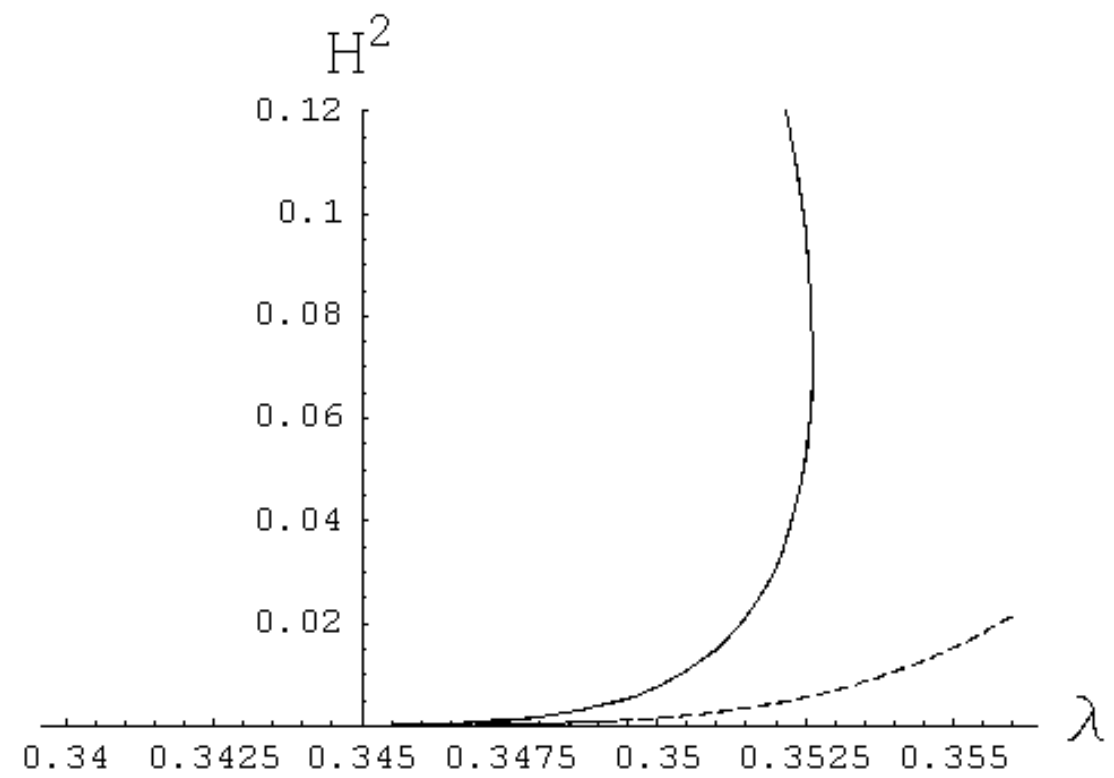

FIG. 2: Closeup of the region $\lambda[t]>1 / 3$. The solid curve is the solution to the Friedman equation. The dashed curve represents Eq. (80).

or decreases. To see what happens, we consider the Friedmann equation in the limit $\lambda[t] \rightarrow 0$

$$
H^{2} \simeq \frac{\lambda[t] \tilde{Q}_{0}}{3 \omega^{2}}
$$

and take the derivative with respect to $\mu$ of this equation to obtain

$$
\frac{\partial H^{2}}{\partial \mu}=-\left(1-\frac{Q_{0} \lambda[t]^{2}}{H^{2} \omega^{2}}\right)^{-1} \frac{2 Q_{0} \lambda[t]^{2}}{\mu \omega^{2}}=-\frac{2 Q_{0} \lambda[t]^{2}}{\mu \omega^{2}}+\mathcal{O}(\lambda[t])^{4}<0
$$

Since this expression is always negative, we find that upon decreasing $\mu, H$ will increase and therefore $\lambda[t]$ will increase. In other words: starting at the origin of figure VB 1, we run along the curve to the right. It is important to realize that we cannot change the running 'direction' on the curve. Also 'stopping' on the curve is not possible as long as we keep decreasing $\mu$.

Depending on the value of $\Lambda_{0}$ (and thus $\lambda_{0}$ )we see that one actually starts with an effective cosmological constant that is somewhat larger then one would expect from the unmodified Friedmann equation (shown by the dashed line in figure VB 1). Continuing along the curve, at a certain point $H$ starts to decrease. In fact, it is clear that $H$ decreases faster and faster until a point where it decreases faster then $\mu$ and therefore eventually $\lambda[t]$ starts to decrease. This must be the case, since this is the only solution to the Friedmann equation in this regime and we see no good reason why the Friedmann equation should break down. In figure 2 we show how $H$ goes to zero, compared to our estimate (80). We see that $H$ decreases even faster then exponentially as $\lambda[t]$ approaches $\lambda_{\text {crit }}=1 / 3$. 
The closer $\lambda[t]$ to $1 / 3$ is, the more accurate (80) becomes.

Notice that the fact that $\lambda$ stays finite for all values of $\mu$ between $H$ and 0 is an important property of our $t$ parameter (57). For other -at first instance sensible- choices of $t$ like $t=\ln \left(M_{x} / \mu\right), \Lambda$ and therefore $\lambda$ will hit the Landau pole become infinite.

2. Does $H^{2}$ stay smaller then $\omega \Lambda$ ?

In calculating the effective potential we assumed that (46) holds, or in the present case, where $|\chi| \leq 1$

$$
H^{2} \ll \omega \Lambda \text {. }
$$

We shall now show that this assumption is indeed justified over the whole range of $\lambda[t]$, independent of the parameters.

First of all notice that the assumptions (175) mean that

$$
\omega \geq \mathcal{O}(10)
$$

Therefore it is clear that if the standard Friedmann equation, $H^{2}=\Lambda / 3$, would be correct, the assumption (83) holds. To see whether this is also true for our modified Friedmann equation, we take the derivative of (76) with respect to $\lambda$

$$
\frac{\partial H^{2}}{\partial \lambda}=\frac{\tilde{Q}_{0} \lambda^{2}}{\omega^{2} H^{2}}(3 \lambda-1) \frac{\partial H^{2}}{\partial \lambda}+\frac{\tilde{Q}_{0}}{6 \omega^{2}}\left(2+6 \lambda-45 \lambda^{2}+6(2-9 \lambda) \lambda L\right),
$$

with $L$ defined in (77). To calculate at which $\lambda H$ is maximal, we put $L$ approximately constant and find

$$
\frac{\partial H^{2}}{\partial \lambda}=0 \quad \rightarrow \quad \lambda=\frac{2 L+1+\sqrt{4 L^{2}+16 L+11}}{3(6 L+5)}
$$

and we substitute this in our Friedmann equation, divided by $\omega \Lambda$ to obtain

$$
\left.\frac{H^{2}}{\omega \Lambda}\right|_{\max } \simeq \frac{(L+1)[2 L(2 L+\sqrt{4 L(L+4)+11}+12)-\sqrt{4 L(L+4)+11}+19]}{3(6 L+5)^{2} \omega} .
$$

Since we know that in this regime $H^{2}=\mathcal{O}(\Lambda)$, it is safe to say that $L$ grows to as most $\mathcal{O}(10)$. With this estimate and (84) we get approximately

$$
\left.\frac{H^{2}}{\omega \Lambda}\right|_{\max } \simeq 0.1
$$

and thus our assumption in (46) is satisfied. 


\section{The infrared sector}

If the mechanism described above is indeed responsible for the small effective cosmological constant measured today, we require that $\mu$ runs at least to a value $\mu_{\min }$ such that $H\left(\mu_{\min }\right)=H_{0}$. Here $H_{0} \simeq 1.5 \times 10^{-42} \mathrm{GeV}$ denotes the Hubble parameter as it is measured today. The analysis presented in this subsection is oversimplified, since we are neglecting matter and radiation contributions in assuming a pure de Sitter space-time. We can estimate $\mu_{\min }$ by inverting the relation (80),

$$
\begin{aligned}
\mu_{\min } & =H_{0} \exp \left(-\frac{4 \pi m_{p}^{2}}{3 \omega^{2} \Lambda_{0}}+\frac{1}{2} \frac{1}{1+\left[2 \ln \left(\frac{8 \pi m_{p}^{2}}{3 \omega H_{0}^{2}}\right)\right]^{-1}}\right) \\
& \simeq H_{0} \exp \left(-\frac{4 \pi m_{p}^{2}}{3 \omega^{2} \Lambda_{0}}+\frac{1}{2}\right) .
\end{aligned}
$$

Notice that the subscript zero for $H$ means 'today', while for $\Lambda$ it means 'at $\mu=0$.

According to the logic in section (IB $)$ the scale (89) corresponds with the largest scale $k$ amplified during primordial cosmic inflation. Since the number of e-folds is given by

$$
N_{\text {tot }}=\ln \left(\frac{H_{I}}{k}\right)
$$

with $H_{I}$ the Hubble parameter during inflation, we get

$$
\begin{aligned}
N_{\mathrm{tot}} & \simeq 60+\ln \left(\frac{H_{0}}{\mu_{\min }}\right) \\
& \simeq 60+\frac{4 \pi m_{p}^{2}}{9 \omega^{2} H_{I}^{2}}
\end{aligned}
$$

where we used the usual estimate that $\ln \left(\frac{H_{I}}{H_{0}}\right) \simeq 60$ and we assumed that $H_{I}^{2} \simeq \Lambda_{0} / 3$. We can rewrite (91) by noting that the power spectrum of curvature perturbation has an amplitude squared,

$$
\Delta_{\mathcal{R}}^{2} \simeq \frac{H_{I}^{2}}{\pi \epsilon m_{p}^{2}} \simeq 2.4 \times 10^{-9},
$$

where $\epsilon$ is the slow roll parameter in inflation, defined as $\epsilon=-\left(d H_{I} / d t\right) / H_{I}^{2}$ (in scalar inflationary models its numerical value is typically of the order $10^{-2}$ ). With this the total number of e-foldings (91) becomes,

$$
N_{\text {tot }} \simeq 60+\frac{4}{9 \omega^{2} \epsilon \Delta_{\mathcal{R}}^{2}} \simeq 60+2 \times 10^{8} \times \frac{1}{\omega^{2} \epsilon} .
$$

This provides an estimate of the duration of inflation in our model, which decreases as $1 / \omega^{2}$ as $\omega$ increases. Even though we are unable to make a direct estimate of the residual dark energy density in our model, in Eq. (93) we have provided a link between the the dark energy density (the Hubble parameter today) and the duration of inflation. 


\section{DISCUSSION AND CONCLUSION}

In this paper we consider the one loop vacuum fluctuations in de Sitter space of a nonsymmetric tensor field, with a mass generated by a coupling to the cosmological constant and the Ricci scalar. While such a field is motivated by nonsymmetric gravitational theories, the present discussion is independent of this fact. We believe that qualitatively the results presented here also hold for e.g. a scalar field whose mass is generated by $\Lambda$. The calculation presented in this work shows that, as one would expect, the coupling constants of the theory $\Lambda, Q=\left(16 \pi G_{N}\right)^{-1}$ and $b$ - which correspond to the coupling constants of the terms $R^{0}, R$ and $R^{2}$ in the gravitational action, respectively - become renormalized. The renormalization depends, of course, on the renormalization scale $\mu$ and after RG improvement we obtain the standard result that the constants (in principle) run logarithmically with $\mu$. Usually this logarithmic running is used to argue that the RG flow cannot drive the cosmological constant to zero. Indeed it seems implausible that such a mild running could explain the smallness of $\Lambda G_{N} \sim 10^{-122}$, which defines the gravitational hierachy problem.

The main thrust of the present work is the observation that the observed, effective $\Lambda_{\text {eff }}$ may dramatically differ from the (RG improved) parameter $\Lambda$. The reason is that $\Lambda_{\text {eff }}$ is given by the Friedmann equation, $H^{2}=\Lambda_{\text {eff }} / 3$, and this equation gets modified by the loop effects. This modification is typically of the order $m^{2} / m_{p}^{2}$, where $m$ is the mass of the field whose contribution we are calculating. This quantity will therefore be in general suppressed. However in our case $m \sim \Lambda$ and standard quantum field theory calculations tell us that $\Lambda$ may be comparable to $m_{p}^{2}$. In other words, a large $\Lambda$ implies substantial modification of the Friedmann equation by loop quantum corrections. At the same time our analysis shows that the Newton constant remains to a good approximation frozen. This is important, since a fast running of the Newton constant could be disastrous in the view of Solar system tests of GR.

Furthermore, we find that as $\mu$ runs towards zero, $H$ - and thus $\Lambda_{\text {eff }}$ - changes mildly for some range of $\mu$. However from a certain value of $\mu, H$ starts to decrase rapidly, eventually even faster then linearly, $H \propto \mu$. Therefore, while $\Lambda$ changes only logarithmically with $\mu, \Lambda_{\text {eff }}$ can decrease as power law. In this manner our model circumvents the usual statement that RG flow cannot relax the cosmological constant. It is important to stress that this is independent of the size of the initial $\Lambda$. The initial conditions influence only at what value of $\mu$ the dramatic decrease in $\Lambda_{\text {eff }}$ begins to take place.

An important point we wish to address now is why do we sent $\mu$ to zero? To understand this, it is first of all necessary to understand that the starting point of our running, $\mu=H$, is in the 
ultraviolet, where both $\Lambda$ and $H$ are of the order the Planck scale $m_{p}$. However in de Sitter space the Hubble parameter is not the lowest possible scale. In fact, many physical modes have wavelengths, that during an inflationary de Sitter phase get amplified and their wavelength grows larger then the hubble length, $H^{-1}$. In order to get a theory that takes proper account of these super-Hubble modes, the infrared $\mu$ should be taken as small as the smallest energy of these modes. Since we are interested in the behavior of the theory on large (cosmological) scales, this is exactly what one should do.

Another interesting question is how applicable our model is to the real world. Strictly speaking, our results are only valid in de Sitter space and one could wonder if our results also apply to, for example, quasi-de Sitter spaces where $H$ is a (mild) function of time. Quasi-de Sitter spaces are relevant for inflationary models and also in the case whenever $\Lambda_{\text {eff }}$ dominates the energy density of the Universe, as it is today. Even though we have performed our analysis in de Sitter space, we expect that our analysis should apply whenever $d H / d t \ll H^{2}$. This condition is satisfied if $\Lambda_{\text {eff }} /\left(8 \pi G_{N}\right)$ dominates the energy density of the Universe, which is also (marginally) true today.

There might be implications of our model for inflation. One could envisage a scenario, where inflation is caused by a large cosmological constant and as times goes on more and more of infrared modes are produced, pushing the relevant RG scale $\mu$ further and further to the infrared, such that $\Lambda_{\text {eff }}$ eventually decreases, terminating inflation, resulting in a standard decelerating Friedmann universe. This type of reasoning has also been pursued in Refs. [39, 40, 41] by using different techniques. An explicit calculation is necessary in the present or similar model however in order to illuminate the questions concerning inflationary dynamics and cosmological perturbations. Based on the assumption that the scale $\mu$ corresponds to the largest scale amplified during inflation, in section $\mathrm{VB}$ we derive a relation between the total number of e-foldings during inflation in our model (driven by a bare cosmological constant) and the dark energy density as measured today. However at the moment we are unable to provide a direct estimate of the dark energy density.

While our model shows interesting behavior, there are some potential problems. First of all one might argue that our model suggests that at small scales $\Lambda_{\text {eff }}$ is huge, and this could present problems to e.g. solar system measurements. However, this reasoning is not correct. Even in small scale experiments, the large scale modes still have their effect on the vacuum. In other words: it is impossible to decouple the infrared modes from the vacuum.

a more serious concern is the validity of the perturbative approach. Indeed in our model tree level and one loop contributions are comparable and therefore it is not clear whether our results hold if one includes higher loop corrections. Furthermore there is a similar problem in the renormalization procedure. The choice of the renormalization scheme and the running parameter $t$ are in principle 
arbitrary. Different choices however differ only at higher loop order. Since higher loop contributions are probably substantial in our model, there is no way of telling which effects are induced by our choices and which effects are really physical.

Despite these problems we do believe that the following is true. A field whose mass is generated by the cosmological constant might generate a huge back-reaction on the vacuum. This back-reaction can be of the same order of magnitude as the original cosmological constant. It might be that the exact structure of the modified Friedmann equation is such that, because of the RG running of $\Lambda$, the effective cosmological constant becomes tiny, without fine tuning and regardless of the initial condition. In this work we have shown an explicit example of this behavior.

\section{ACKNOWLEDGEMENT}

We would like to thank Frank Saueressig for sharing his insights in the renormalization group.

\section{APPENDIX A: SEPARATING THE FINITE AND INFINITE PARTS OF $\Gamma_{B}$}

To separate the infinite from the finite part of the effective action $\Gamma$ (42), we use the following expression

$\frac{\Gamma\left(\frac{D-1}{2}+\nu_{n}\right) \Gamma\left(\frac{D-1}{2}-\nu_{n}\right)}{\Gamma\left(\frac{1}{2}+\nu_{n}\right) \Gamma\left(\frac{1}{2}-\nu_{n}\right)}=\left(\left(\frac{D-3}{2}\right)^{2}-\nu_{n}^{2}\right)\left[1+\frac{D-4}{2}\left(\psi\left(\frac{1}{2}+\tilde{\nu}_{n}\right)+\psi\left(\frac{1}{2}-\tilde{\nu}_{n}\right)\right)\right]+\mathcal{O}\left((D-4)^{2}\right)$,

where $\tilde{\nu}_{n}=\left.\nu_{n}\right|_{D=4}$ and $\psi(z)$ is the digamma function, defined by

$$
\psi(z) \equiv \frac{\Gamma^{\prime}(z)}{\Gamma(z)} .
$$

\section{Evaluating $\Gamma_{w}$}

By making use of Eqs. (42) and (A1) we can break $\Gamma_{w}$ into the infinite and finite parts as follows:

$$
\begin{aligned}
\Gamma_{w}=- & \int_{x} \sqrt{-g}\left[\int d w \frac{D-1}{4(4 \pi)^{D / 2}} H^{D} \Gamma(1-D / 2)((D-2)(D-4+w)-(2(D-5)+w))\right. \\
& \left.+\frac{3 H^{4}}{64 \pi^{2}} \int d w\left(2 w\left(\psi\left(1 / 2+\tilde{\nu}_{2}\right)+\psi\left(1 / 2-\tilde{\nu}_{2}\right)\right)-(w-2)\left(\psi\left(1 / 2+\tilde{\nu}_{3}\right)+\psi\left(1 / 2-\tilde{\nu}_{3}\right)\right)\right)\right] .
\end{aligned}
$$

This breakdown is not unique (since we can always shift a finite part of the infinite part back and forth), but when taken together with the counterterms, this nonuniqueness has no physical 
consequence. The integral in the infinite part is easy to evaluate:

$$
\begin{aligned}
\Gamma_{w-i n f}=-\int_{x} \sqrt{-g}\left[\frac{D-1}{4(4 \pi)^{D / 2}} \Gamma(1-D / 2)\right. & \frac{1}{2}(D-3)(\omega \Lambda)^{2} H^{D-4} \\
& +\left(D^{2}-8 D+18+(D-3) \chi\right) \omega \Lambda H^{D-2} \\
& \left.\left.+\left(\left(D^{2}-8 D+18\right) \chi+\frac{1}{2}(D-3) \chi^{2}\right) H^{D}\right)\right],
\end{aligned}
$$

where we used that (27)

$$
m^{2}=\omega \Lambda+\chi H^{2} .
$$

To evaluate the finite part we assume that

$$
w=\frac{\omega \Lambda+\chi H^{2}}{H^{2}} \gg 1,
$$

which we justify in section $\mathrm{VB} 2$. With this assumption we can use

$$
\begin{aligned}
& \psi\left(1 / 2+\nu_{2}\right)+\psi\left(1 / 2-\nu_{2}\right)=\ln (w)-\frac{1}{3 w}-\frac{1}{15 w^{2}}+\mathcal{O}\left(w^{-3}\right) \\
& \psi\left(1 / 2+\nu_{3}\right)+\psi\left(1 / 2-\nu_{3}\right)=\ln (w)-\frac{7}{3 w}-\frac{41}{15 w^{2}}+\mathcal{O}\left(w^{-3}\right)
\end{aligned}
$$

and find:

$$
\begin{aligned}
& \Gamma_{w-f i n}=-\int_{x} \sqrt{-g} \frac{3}{64 \pi^{2}} H^{4} {\left[-\frac{31}{15} \ln (w)+\left(-\frac{1}{3}+2 \ln (w)\right) w+\left(-\frac{1}{4}+\frac{1}{2} \ln (w)\right) w^{2}\right] } \\
&=-\int_{x} \sqrt{-g} \frac{3}{63 \pi^{2}}\left[-\frac{31}{15} H^{4} \ln (w)+\left(\frac{(\omega \Lambda)^{2}}{2}+H^{2} \omega \Lambda(2+\chi)+H^{4} \chi\left(2+\frac{\chi}{2}\right)\right) \ln (w)\right. \\
&\left.-\frac{(\omega \Lambda)^{2}}{4}-H^{2} \omega \Lambda\left(\frac{1}{3}+\frac{\chi}{2}\right)-H^{4} \chi\left(\frac{1}{3}+\frac{\chi}{4}\right)\right]
\end{aligned}
$$

\section{Evaluating $\Gamma_{H}$}

From Eq. (42) we can write

$$
\begin{aligned}
\Gamma_{H}= & -\int_{x} \sqrt{-g}[ \\
& \int d H^{2}(D-1) \frac{H^{D-2}}{4(4 \pi)^{D / 2}} \Gamma(1-D / 2)((D-2)(D-4+w) w+(D-6-w)(2(D-5)+w)) \\
& \left.+\frac{3}{64 \pi^{2}} \int d H^{2} H^{D-2}\left(2 w^{2}\left(\psi\left(1 / 2+\tilde{\nu}_{2}\right)+\psi\left(1 / 2-\tilde{\nu}_{2}\right)\right)-\left(w^{2}-4\right)\left(\psi\left(1 / 2+\tilde{\nu}_{3}\right)+\psi\left(1 / 2-\tilde{\nu}_{3}\right)\right)\right)\right] .
\end{aligned}
$$


We start with the infinite part:

$$
\begin{aligned}
\Gamma_{H-i n f}=-\int_{x} \sqrt{-g}\left[\frac{D-1}{4(4 \pi)^{D / 2}} \frac{2}{D} H^{D} \Gamma(1-D / 2)\left(2(D-5)(D-6)+(D-4)(D-3) w+(D-3) w^{2}\right)\right] \\
=-\int_{x} \sqrt{-g}\left[\frac { D - 1 } { 4 ( 4 \pi ) ^ { D / 2 } } \frac { 2 } { D } \left(H^{D}\left(2(D-5)(D-6)+(D-4)(D-3) \chi+(D-3) \chi^{2}\right)\right.\right. \\
\left.\left.+H^{D-2} \omega \Lambda((D-4)(D-3)+2(D-3) \chi)+H^{D-4}(\omega \Lambda)^{2}(D-3)\right)\right] .
\end{aligned}
$$

The finite part is easily evaluated and gives

$$
\begin{aligned}
& \Gamma_{H-f i n}=-\int_{x} \sqrt{-g} \frac{3}{64 \pi^{2}} H^{4}\left(2 \ln (w)+\frac{w^{2}}{2} \ln (w)+\frac{5}{6} w+\frac{13}{10}\right) \\
& \quad=-\int_{x} \sqrt{-g} \frac{3}{64 \pi^{2}}\left(2 H^{4} \ln (w)+\left(\frac{(\omega \Lambda)^{2}}{2}+\chi \omega \Lambda H^{2}+\frac{1}{2} \chi^{2} H^{4}\right) \ln (w)+\frac{13}{10} H^{4}+\frac{5}{6}\left(\chi H^{4}+\omega \Lambda H^{2}\right)\right) .
\end{aligned}
$$

\section{Combining the results}

Since in $D=4$ our counterterms are of the form $R^{0}, R^{1}, R^{2} \ldots$, we need to expand the $D$ dependent

powers in $H$. To achieve this, one needs to introduce an arbitrary mass scale $\mu$, as can be seen from the following identities:

$$
\begin{aligned}
H^{D} & =\mu^{D-4}\left(H^{4}+\frac{D-4}{2} H^{4} \ln \left(\frac{H^{2}}{\mu^{2}}\right)\right)+\mathcal{O}\left((D-4)^{2}\right) \\
H^{D-2} \omega \Lambda & =\mu^{D-4}\left(H^{2} \omega \Lambda+\frac{D-4}{2} H^{2} \omega \Lambda \ln \left(\frac{H^{2}}{\mu^{2}}\right)\right)+\mathcal{O}\left((D-4)^{2}\right) \\
H^{D-4}(\omega \Lambda)^{2} & =\mu^{D-4}\left((\omega \Lambda)^{2}+\frac{D-4}{2}(\omega \Lambda)^{2} \ln \left(\frac{H^{2}}{\mu^{2}}\right)\right)+\mathcal{O}\left((D-4)^{2}\right) .
\end{aligned}
$$

Notice that this statement is equivalent to the argument used in e.g. [12], where $\mu$ is introduced in order to give physical constants the right dimensionality. Using (A10), we combine Eqs. (A3), (A7), 
(A8) and (A9) to obtain our final result for the 1-loop effective action for the $B$-field

$$
\begin{aligned}
\Gamma_{B}= & -\int_{x} \sqrt{-g}[ \\
& \frac{D-1}{4(4 \pi)^{D / 2}} \Gamma(1-D / 2)\left(H^{4} \frac{1}{2 D}\left[8(D-5)(D-6)+2\left(24+D\left(D^{2}-6 D+4\right)\right) \chi+(D-3)(D+4) \chi^{2}\right]\right. \\
& \left.\quad+H^{2} \omega \Lambda\left(4+D^{2}+D(\chi-6)-\frac{12(\chi-2)}{D}+\chi\right)+(\omega \Lambda)^{2} \frac{(D-3)(D+4)}{2 D}\right) \mu^{D-4} \\
& +\frac{3}{64 \pi^{2}}\left(\left((\omega \Lambda)^{2}+2(1+\chi) \omega \Lambda H^{2}+2\left(1+\chi+\frac{\chi^{2}}{2}\right) H^{4}\right) \ln \left(\frac{\omega \Lambda+\chi H^{2}}{\mu^{2}}\right)-\frac{31}{15} H^{4} \ln \left(\frac{\omega \Lambda+\chi H^{2}}{H^{2}}\right)\right. \\
& \left.\left.\quad-\frac{(\omega \Lambda)^{2}}{4}+\frac{H^{2} \omega \Lambda}{2}(1-\chi)+\frac{\chi H^{4}}{2}\left(1-\frac{\chi}{2}\right)+\frac{13}{10} H^{4}\right)\right]+\mathcal{O}\left(H^{4} \frac{H^{2}}{m^{2}}\right) \\
\equiv & \int_{x} \delta \mathcal{L}
\end{aligned}
$$

\section{APPENDIX B: METHOD OF CHARACTERISTICS}

In this Appendix we shall describe how the method of characteristics works [37] [38]. The requirement that physics is independent of $\mu$, is equivalent to requiring that the theory is invariant under the transformation

$$
\mu \rightarrow \mu \mathrm{e}^{t} \equiv \bar{\mu}[t]
$$

with $t$ a real number. Since our coupling parameters $\Lambda, Q$ and $b$ depend on $\mu$, the transformation (B1) means that

$$
\begin{aligned}
\Lambda & \rightarrow \bar{\Lambda}[t] \\
Q & \rightarrow \bar{Q}[t] \\
b & \rightarrow \bar{b}[t] .
\end{aligned}
$$

Quantities that we require to be independent of $\mu$, like the effective action, need to be independent of this transformation and therefore

$$
\Gamma(\Lambda, Q, \mu)=\Gamma(\bar{\Lambda}[t], \bar{Q}[t], \bar{b}[t], \bar{\mu}[t])
$$

This can only be true if

$$
\begin{aligned}
0 & =\frac{d}{d t} \Gamma(\bar{\Lambda}[t], \bar{Q}[t], \bar{b}[t], \bar{\mu}[t]) \\
& =\left(\bar{\mu} \frac{\partial}{\partial \bar{\mu}}+\frac{d \bar{\Lambda}}{d t} \frac{\partial}{\partial \bar{\Lambda}}+\frac{d \bar{Q}}{d t} \frac{\partial}{\partial \bar{Q}}+\frac{d \bar{b}}{d t} \frac{\partial}{\partial \bar{b}}\right) \Gamma(\bar{\Lambda}[t], \bar{Q}[t], \bar{b}[t], \bar{\mu}[t])
\end{aligned}
$$


Comparing this with the Callan-Symanzik equation

$$
\left(\mu \frac{\partial}{\partial \mu}+\beta_{\Lambda} \frac{\partial}{\partial \Lambda}+\beta_{Q} \frac{\partial}{\partial Q}+\beta_{b} \frac{\partial}{\partial \bar{b}}\right) \Gamma(\Lambda, Q, b, \mu)=0
$$

we conclude that the transformation (B1) and (B2), with parameters given by

$$
\begin{aligned}
\frac{d \bar{\Lambda}[t]}{d t} & =\beta_{\Lambda}[\bar{\Lambda}[t], \bar{Q}[t]] & \bar{\Lambda}[0] & =\Lambda \\
\frac{d \bar{Q}[t]}{d t} & =\beta_{Q}[\bar{\Lambda}[t]] & \bar{Q}[0] & =Q \\
\frac{d \bar{b}[t]}{d t} & =\beta_{b} & \bar{b}[0] & =b
\end{aligned}
$$

enforces the Callan-Symanzik equation for the effective action $\Gamma$. 
[1] S. Weinberg, "The cosmological constant problem," Rev. Mod. Phys. 61 (1989) 1-23.

[2] S. Nobbenhuis, "The cosmological constant problem, an inspiration for new physics," gr-qc/0609011.

[3] S. K. Lamoreaux, "Demonstration of the Casimir force in the 0.6 to 6 micrometers range," Phys. Rev. Lett. 78 (1997) 5-7.

[4] H. B. G. Casimir, "On the attraction between two perfectly conducting plates," Kon. Ned. Akad. Wetensch. Proc. 51 (1948) 793-795.

[5] R. L. Jaffe, "The Casimir effect and the quantum vacuum," Phys. Rev. D72 (2005) 021301, hep-th/0503158.

[6] Supernova Cosmology Project Collaboration, S. Perlmutter et al., "Measurements of Omega and Lambda from 42 High-Redshift Supernovae," Astrophys. J. 517 (1999) 565-586, astro-ph/9812133.

[7] Supernova Search Team Collaboration, A. G. Riess et al., "Observational Evidence from Supernovae for an Accelerating Universe and a Cosmological Constant," Astron. J. 116 (1998) 1009-1038, astro-ph/9805201.

[8] P. Astier et al., "The Supernova Legacy Survey: Measurement of Omega O Omega $_{L}$ ambda and w from the First Year Data Set," Astron. Astrophys. 447 (2006) 31-48, astro-ph/0510447.

[9] Supernova Search Team Collaboration, A. G. Riess et al., "Type Ia Supernova Discoveries at z¿1 From the Hubble Space Telescope: Evidence for Past Deceleration and Constraints on Dark Energy Evolution," Astrophys. J. 607 (2004) 665-687, astro-ph/0402512.

[10] D. N. Spergel et al., "Wilkinson Microwave Anisotropy Probe (WMAP) three year results: Implications for cosmology," astro-ph/0603449.

[11] M. E. Peskin and D. V. Schroeder, "An Introduction to quantum field theory," Reading, USA: Addison-Wesley (1995) 842 p.

[12] C. Itzykson and J. B. Zuber, "Quantum field theory,". New York, Usa: Mcgraw-hill (1980) 705 P.(International Series In Pure and Applied Physics).

[13] I. L. Shapiro and J. Sola, "On the scaling behavior of the cosmological constant and the possible existence of new forces and new light degrees of freedom," Phys. Lett. B475 (2000) 236-246, hep-ph/9910462.

[14] I. L. Shapiro and J. Sola, "Scaling behavior of the cosmological constant: Interface between quantum field theory and cosmology," JHEP 02 (2002) 006, hep-th/0012227.

[15] I. L. Shapiro and J. Sola, "Cosmological constant problems and renormalization group," 
gr-qc/0611055.

[16] M. Reuter, "Nonperturbative Evolution Equation for Quantum Gravity," Phys. Rev. D57 (1998) 971-985, hep-th/9605030

[17] M. Reuter and F. Saueressig, "Renormalization group flow of quantum gravity in the Einstein-Hilbert truncation," Phys. Rev. D65 (2002) 065016, hep-th/0110054.

[18] M. Reuter and H. Weyer, "Quantum gravity at astrophysical distances?," JCAP 0412 (2004) 001, hep-th/0410119.

[19] T. Prokopec, "A solution to the cosmological constant problem," gr-qc/0603088.

[20] J. W. Moffat, "Nonsymmetric gravitational theory," Phys. Lett. B355 (1995) 447-452, gr-qc/9411006

[21] M. A. Clayton, "Massive NGT and spherically symmetric systems," J. Math. Phys. 37 (1996) $395-420$, gr-qc/9505005.

[22] T. Janssen and T. Prokopec, "Problems and hopes in nonsymmetric gravity," gr-qc/0611005.

[23] Y. Mao, M. Tegmark, A. Guth, and S. Cabi, "Constraining Torsion with Gravity Probe B," gr-qc/0608121.

[24] T. Prokopec and W. Valkenburg, "Antisymmetric Metric Field as Dark Matter," astro-ph/0606315.

[25] T. Prokopec and W. Valkenburg, "The cosmology of the nonsymmetric theory of gravitation," Phys. Lett. B636 (2006) 1-4, astro-ph/0503289.

[26] W. Valkenburg, "Linearized nonsymmetric metric pertubations in cosmology," master's thesis at the ITP of Utecht University, Available at http://www1.phys.uu.nl/wwwitf/teaching/thesis.htm (2006).

[27] T. Janssen and T. Prokopec, "Instabilities in the nonsymmetric theory of gravitation," Class. Quant. Grav. 23 (2006) 4967-4982, gr-qc/0604094.

[28] E. O. Kahya and R. P. Woodard, "Charged scalar self-mass during inflation," Phys. Rev. D72 (2005) 104001, gr-qc/0508015.

[29] N. C. Tsamis and R. P. Woodard, "A maximally symmetric vector propagator," gr-qc/0608069.

[30] M. Spradlin, A. Strominger, and A. Volovich, "Les Houches lectures on de Sitter space," hep-th/0110007.

[31] B. Allen, "Vacuum states in de Sitter space," Phys. Rev. D32 (1985) 3136.

[32] B. Allen and A. Folacci, "The massless minimally coupled scalar field in de Sitter space," Phys. Rev. D35 (1987) 3771.

[33] N. A. Chernikov and E. A. Tagirov, "Quantum theory of scalar fields in de Sitter space-time," Annales Poincare Phys. Theor. A9 (1968) 109. 
[34] N. C. Tsamis and R. P. Woodard, "Dimensionally regulated graviton 1-point function in de Sitter," Annals Phys. 321 (2006) 875-893, gr-qc/0506056.

[35] N. D. Birrell and P. C. W. Davies, "Quantum fields in curved space,". Cambridge, Uk: Univ. Pr. ( 1982) 340p.

[36] S. R. Coleman and E. Weinberg, "Radiative corrections as the origin of spontaneous symmetry breaking," Phys. Rev. D7 (1973) 1888-1910.

[37] H. Kleinert and V. Schulte-Frohlinde, "Critical properties of phi**4-theories,". River Edge, USA: World Scientific (2001) 489 p.

[38] M. Faux, "Notes on renormalization,". Available at http://www.math.columbia.edu/ faux/geometry/renormalization.ps.

[39] N. C. Tsamis and R. P. Woodard, "Quantum Gravity Slows Inflation," Nucl. Phys. B474 (1996) 235-248, hep-ph/9602315.

[40] N. C. Tsamis and R. P. Woodard, "The quantum gravitational back-reaction on inflation," Annals Phys. 253 (1997) 1-54, hep-ph/9602316.

[41] L. R. W. Abramo, R. H. Brandenberger, and V. F. Mukhanov, "The energy-momentum tensor for cosmological perturbations," Phys. Rev. D56 (1997) 3248-3257, gr-qc/9704037.

[42] E. Mottola, "Particle creation in de Sitter space," Phys. Rev. D31 (1985) 754.

[43] H. G. Embacher, G. Grubl, and R. Patek, "Gauge invariant energy momentum tensor for massive QED," Phys. Rev. D33 (1986) 1162-1165.

[44] H. Ruegg and M. Ruiz-Altaba, "The Stueckelberg field," Int. J. Mod. Phys. A19 (2004) 3265-3348, hep-th/0304245.

[45] $\alpha$-vacua are probably unphysical since the propagator equation requires an additional $\delta$-function source at the antipodal point [31] 30] [42], henceforth we shall not consider them in this paper.

[46] The terminology 'gauge fixing' is a bit misleading, since the theory has no explicit gauge invariance. However, one still is able to add a 'gauge fixing' term. It plays the rôle of a Lagrange multiplier, imposing the Lorentz condition. See e.g [43] [44].

[47] Numerical analysis shows that $\lambda_{\text {crit }}$ is marginally larger then $1 / 3$. 